\title{
Determinação de Códigos Convolucionais de Memória Unitária Ótimos: um Problema de Otimização via Seleção e Alocação de Recursos
}

\author{
Amir Said e Reginaldo Palazzo Jr.
}

\begin{abstract}
Neste trabalho é apresentado um procedimento via programação mátemática, em direção a uma sistematizaçāo do problema de determinaçăo de códigos convolucionais ótimos, especificamente aqueles pertencentes à classe de memória unitária. Este procedimento utiliza técnicas típicas de equivalência e redução de problemas, comumente empregadas na análise de complexidade e intratabilidade, e culmina com o estabelecimento de dois problemas de otimização, um de seleção e outro de alocação de recursos. A solução destes problemas consiste na determinação dos tipos de colunas das submatrizes geradoras bem como na sua ordenção correta. Por fim, são apresentados novos códigos convolucionais de memória unitária, obtidos através deste novo procedimento.
\end{abstract}

\section{Introdução}

Q problema de determinação de códigos convolucionais ótimos a partir de propriedades algébricas ou modelos matemáticos advém desde a proposta, feita por Elias [1] em 1955, desta classe de códigos. Um passo importante nesta direção foi dado por Viterbi [2] em 1967, quando do estabelecimento do algoritmo que hoje leva o seu nome que, posteriormente, provou-se ser equivalente à aplicação da programação dinâmica ao problema de decodificação por máxima verossimilhança. No ano seguinte, Odenwalder [3] foi o primeiro que efetivamente apresentou códigos convolucionais segundo o algoritmo apresentado em [2]. Desde então, muitos pesquisadores têm apresentado novos códigos convolucionais através de algoritmos que são aprimoramentos da adaptação do algoritmo de Viterbi à seleção do código ótimo, via busca exaustiva das matrizes geradoras. Dentro desta categoria encontramos os algoritmos propostos por Larsen [4], Paaske [5], Johannesson e Paaske [6], Massey e Costello [7] e Daut et alli [8].

Os autores săo professores da Faculdade de Engenharia Elêtrica da UNICAMP, Caixa Postal 6101, 13081, Campinas, SP. 
Um novo avanço foi dado por Lee [9] em 1976, quando da proposta de códigos convolucionais de memória unitária. Embora não apresentando uma estrutura algébrica equivalente aos códigos de bloco, a conexão foi feita considerando-se a truncagem do código convolucional com relação ao primeiro comprimento de restrição, dando origem a um código de bloco equivalente. Mostrou-se que todos os códigos convolucionais podem ser enquadrados na classe dos códigos convolucionais de memória unitária, o que garante que os códigos ótimos encontrados nesta categoria, em relação à capacidade de correção de erros, não são inferiores aos pertencentes a outras categorias. Além disto, Lee mostrou que o uso de memória unitária (que, de certo modo, é equivalente a se operar em bytes ao invés de bits) permite melhorar sensivelmente a capacidade de correção dos códigos convolucionais, sem aumentar a complexidade na decodificação pelo algoritmo de Viterbi.

Entretanto, em 1983, uma proposta de equivalência do problema de determinação de códigos convolucionais com o problema de máximo fluxo em redes, apresentada em [10], possibilitou a introdução de novas técnicas com estrutura matemática objetivando a sistematização da resolução do problema em questão. Como resultado, uma quantidade considerável de novos códigos convolucionais foi apresentada. No entanto, tais coçdigos estão restritos a um comprimento de restrição igual a 10 para taxas $1 / n$ e até 5 para taxa $b / n(2 \leqslant b$ $\leqslant 5)$.

A principal contribuição deste trabalho é a apresentação de uma formulação matemática do problema de determinação de códigos convolucionais ótimos, até então inexistente. Desta forma procuramos estender de forma consistente as idéias e resultados apresentados em [9] e [10], adaptados para códigos convolucionais de memória unitária. É definido um novo procedimento, idealizado a partir de processos de equivalência e redução do problema, que conduz à sua decomposição em dois subproblemas de otimização, a saber, um de seleção e um de alocação de recursos. Como resultado, temos a capacidade de obter novos códigos convolucionais binários de memória unitária ótimos.

$\mathrm{Na}$ Seção 2, os processos de equivalência e redução são apresentados. $\mathrm{Na}$ Seção 3 , demonstra-se como o problema original pode ser decomposto e é descrita a correspondente formulação matemática. Na Seção 4, mostra-se a resolução do primeiro subproblema (seleção) e, na Seção 5, a resolução do segundo subproblema (alocação). Os resultados computacionais correspondentes são apresentados na Seção 6. 


\section{Equivalência e Redução}

Em geral, a solução de problemas complexos passa por uma caracterização de equivalência, seguida por um processo de redução. Este procedimento tem por objetivo buscar uma solução do problema original via equivalência, uma vez que o problema reduzido dispōe de uma estrutura matemática bem definida. Este procedimento foi utilizado em [10] com o objetivo de sistematizar a determinação de códigos convolucionais específicos, bem como classes de códigos convolucionais invariantes e variantes no tempo, pois a falta de uma estrutura algébrica acarreta dificuldades substanciais na resolução deste problema. Vários algoritmos foram propostos ao longo dos últimos 18 anos, objetivando fornecer códigos convolucionais ótimos segundo alguns critérios convenientes, principalmente a maximização da distância livre, que é bastante próxima da maximização da capacidade de correção. Porém, a complexidade inerente ao problema não permitiu ainda uma solução fechada.

O codificador convolucional pode ser visto como uma máquina de estados finitos. Sua representação matemática é dada pela quintupla $(S, 1, O, p, q)$, onde $S$ é o conjunto de estados; I é o conjunto de entradas; $O$ é o conjunto de saídas; $\mathrm{p}: \mathrm{IxS} \rightarrow \mathrm{S}$ e q: IxS $\rightarrow$ O são funçōes que determinam a transição de estado e saída, respectivamente. Nesta seçāo estaremos interessados em focalizar a atenção nos processos de equivalência e redução mencionados. A equivalência do problema de determinação de um código convolucional especiffico com o problema de determinação do fluxo máximo em redes depende fortemente da conjetura a ser apresentada. Antes, porém, de estabelecê-la, iremos, através de um exemplo, explicitar esta equivalência. Caracterizaremos um código convolucional através da tripla $(n, b, m)$, onde $n$ é o comprimento da palavra código ramo, b é o comprimento dos dados de entrada e $m$ é o número de memórias. Tal código tem taxa $r=b / n$. A Fig. 1 (a) mostra

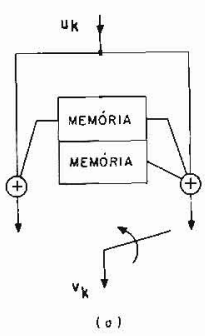

$u_{k}$ - dados de entrada $v_{k}$ - saida codificada
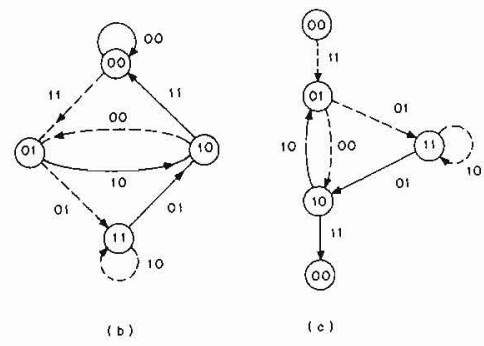

$a b$ - estado $a b$

xy - bits da saída codificada

Figura 1. Representações de um codificador convolucional. 
a representação esquemática de um codificador binário com parâmetros $m=2$ e $r=1 / 2$. Na mesma figura temos os correspondentes diagrama de estados (b) e diagrama de estados particionado (c).

Note que, a partir da Fig. 1(c), podemos iniciar o processo de equivalência fazendo as associações entre as variáveis dos problemas equivalentes apresentadas na Tabela 1.

(a)

$$
\begin{gathered}
\text { diagrama de estados } \\
\text { particionado }
\end{gathered} \begin{gathered}
\text { rede de fluxos } \\
\text { capacitada }
\end{gathered}
$$

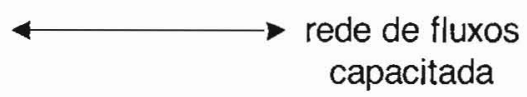

(b) peso de Hamming da palavra gerada na transição i, j

$\left(\omega_{i j}\right)$

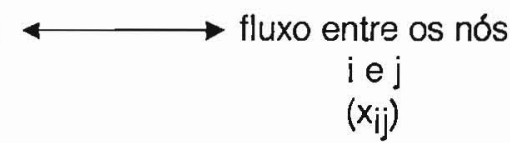

(c) soma dos pesos das palavras entrando e saindo de cada estado fluxo total entrando e saindo de cada nó

(d) distância livre do código $d_{\text {free }}$ máximo fluxo na rede

Tabela 1. Equivalência de problemas.

A associação (c) nada mais é do que a constatação da propriedade de conservação de fluxo, enquanto que a associação (d) é o critério de otimalidade a ser utilizado. Como a distância livre do código é o fator considerado mais importante para minimizar a probabilidade de erro na decodificação [2], a maximização deste parâmetro ế usada para definir a otimalidade do codificador.

Como o codificador convolucional é um sistema linear discreto no tempo (para simplificar, supomos também invariante no tempo), o diagrama de estados pode ser utilizado para a obtenção das equações de estado e de salda do sistema linear associado. Representando por $\mathbf{S}(k)$ o vetor de estados (nós intermediários); por $\mathbf{i}(k)$ o vetor de entrada; por $\mathbf{O}(k)$ o vetor de saída; por $\overline{\mathbf{P}}$ e $\overline{\mathbf{Q}}$ as matrizes de transição de estado; e finalmente por $\overline{\mathbf{R}}$ e $\overline{\mathbf{S}}$ as matrizes de formação da saída, resultam as equações de estado e de saída

$$
\begin{aligned}
& \mathbf{s}(k+1)=\bar{P} \mathbf{s}(k)+\overline{\mathbf{Q}} \mathrm{i}(k) \\
& \mathbf{o}(k)=\overline{\mathrm{R}} \mathbf{s}(k)+\overline{\mathrm{S}} \mathrm{i}(\mathrm{k})
\end{aligned}
$$


Para um codificador binărio de memória unitária, por exemplo, temos a matriz $\overline{\mathbf{P}}$ nula, $\overline{\mathbf{Q}}$ igual à identidade, $\overline{\mathrm{S}}=\mathrm{G}_{\mathbf{0}}$ e $\mathrm{R}=\mathrm{G}_{1}$, onde $\mathrm{G}_{0}$ e $\mathrm{G}_{1}$ são matrizes binárias e as operações em (1) e (2) são definidas em GF(2). Veremos melhor esta classe de códigos posteriormente.

A matriz $\overline{\mathbf{P}}$ é a matriz de transição incorporando apenas os estados intermediários do diagrama de estados particionado. Chamaremos de $\bar{A}$ a matriz de transição aumentada, que difere de $\overline{\mathbf{P}}$ pela inclusão dos estados inicial e final e chamaremos de ã $\tilde{a}_{i j}$ aos elementos de $\bar{A}$. Sejam ainda $L_{i}(\overline{\mathbf{A}})$ e $C_{j}(\bar{A})$ os valores resultantes do produto dos elementos da i-ésima linha e j-ésima coluna de $\overline{\mathrm{A}}$, isto é

$$
L_{i}(\tilde{\mathbf{A}})=\prod_{j=1}^{g} \tilde{a}_{i j}
$$

e

$$
C_{j}(\tilde{A})=\prod_{i=1}^{g} \quad a_{i j}
$$

onde g é o número de estados.

A descrição da rede de fluxo pode assim ser realizada. Seja $\mathbf{G}=(\mathbf{N}, \mathbf{R})$ um grafo direcionado onde $\mathbf{N}$ é um conjunto finito de elementos denominados nós ou estados, isto é, $\mathbf{N}=\{1,2, \ldots, \mathrm{g}\}$ e $\mathbf{R}$ um conjunto também finito de pares ordenados $(i, j)$ denominados ramos. isto é, $R=\{(i, j): i, j \in \mathbf{N}\}$. Seja a capacidade $c_{i j}$ do conjunto $\mathbf{N}$, os elementos 1 e $\mathrm{g}$ serão designados respectivamente nós origem $e$ destino e os demais elementos serão designados por nós intermediårios.

Seja $x_{i j}\left(0 \leqslant x_{i j} \leqslant c_{i j}\right)$ o fluxo do ramo capacitado $(i, j)$ do grafo $G$. A descrição matemática da conservação dos fluxos numa rede é dada por

$$
\sum_{\mathrm{i}} x_{\mathrm{ij}}-\sum_{\mathrm{k}} x_{\mathrm{jk}}=\left\{\begin{array}{l}
-\varphi, \text { se } \mathrm{j}=1 \\
0, \text { se } \mathrm{j} \neq 1 \text { e j } \neq \mathrm{g} \\
\varphi, \text { se } \mathrm{j}=\mathrm{g}
\end{array}\right.
$$

A interpretação de (3) pode ser resumida conforme descrito a seguir. Se $\mathrm{j}=1$, então o primeiro somatório é zero pois não existem fluxos de ramo chegando ao nó 1 e o segundo somatório fornece a soma de todos os fluxos saindo do nó 1 . Se $\mathrm{j}=\mathrm{g}$, só teremos o primeiro somatório visto que o segundo fornece a soma de todos os fluxos de ramo saindo do destino e esta soma é igual a zero. 
Por outro lado, se $j \neq 1$ e $j \neq g$, então ambos os somatórios resultam no mesmo valor de fluxo total saindo ou entrando em cada nó da rede. Quando isto acontece, diz-se que a rede é limitada e balanceada.

A afirmação feita acima para os casos $\mathrm{j}=1 \mathrm{e} \mathrm{j}=\mathrm{g}$, de que o somatório dos fluxos de ramo iguala ao máximo fluxo da rede, está baseada no Teorema de Máximo Fluxo - Mínimo Corte [13] que, por sua vez, estabelece que o fluxo máximo em uma rede é igual à soma dos fluxos nos ramos pertencentes ao mínimo conjunto de corte.

Com esses elementos em mãos podemos estabelecer a seguinte conjetura [10].

\section{Conjetura}

Se existir um codificador convolucional invariante no tempo não sistemático com parâmetros $m$ e $r=b / n$ tal que

$$
L_{i}(\overline{\mathbf{A}})=C_{j}(\overline{\mathbf{A}})=D^{\varphi} \quad \text { para todo } i, j \in \mathbf{N}
$$

(onde D é a função de Bhattacharyya) então o código é dito ótimo.

Dessia forma, a determinação dos códigos convolucionais ótimos não sistemáticos e específicos pode ser caracterizada como um problema de fluxo máximo em redes. Uma vez que a conjetura é assumida verdadeira, o primeiro passo na solução do problema será a determinação do fluxo máximo como função dos parâmetros do código. Assim, temos o teorema que se segue [11].

\section{Teorema 1}

O fluxo máximo para códigos convolucionais não sistemáticos, especificos e invariantes no tempo sobre GF(2), com memória $m$ e taxa $r=b / n$, é dado por

$$
\varphi=n 2^{b-1}
$$

A redução do problema original passa a ser descrita matematicamente como uma rede onde todos os fluxos satisfazem às condições de otimalidade do problema $\mathbf{P}_{1}$, sendo $\omega_{\mathrm{ij}}$ o peso de Hamming do ramo $(\mathrm{i}, \mathrm{j})$ :

6 
$P_{1} \quad$ Maximizar $\sum_{j \in \Omega} \omega_{i j}$

sujeito a

$$
\sum_{i} \omega_{i j}-\sum_{k} \omega_{j k}=\left\{\begin{array}{l}
-\varphi, \text { se } j=1 \\
0, \text { se } j \neq 1 \text { ej } \neq g \\
\varphi, \text { se } j=g
\end{array}\right.
$$

onde $\Omega$ é o conjunto de nós intermediários.

Esta redução é característica de problemas combinatoriais que, sob certas condições, podem ser facilmente solucionados. No caso mais geral, este problema combinatorial ("shortest weight-constrained path") \&́ do tipo NP-completo, sendo do tipo $\mathrm{P}$ (polinomial) se, ao truncarmos a treliça em $\mathrm{k}$ intervalos de tempo, todas as comparaçóes entre pares de palavras código resultantes apresentarem o mesmo peso ou todos os comprimentos forem iguais. Entretanto, podemos aplicar o processo de redução através do estabelecimento de alguns parâmetros que excluirão, numa primeira aproximação, um grande número de soluções factíveis. Um desses parâmetros é a distância mínima do código. Dessa forma, temos o seguinte teorema [11].

\section{Teorema 2}

Para qualquer código convolucional sobre o $\mathrm{GF}(\mathrm{q})$ com memória $\mathrm{m}$, taxa $\mathrm{r}$ $=b / n$ e mdc $(b, n)=1$, a distância mínima é limitada superiormente por.

Caso 1: $b=1$ e $q=2$ (conjunto de corte unitário)

$d_{\min } \leqslant\left[\frac{2^{p_{-1}^{*}}}{2^{p^{*}}-1}\right]\left(p^{*}+m\right) \varphi$

Caso 2: a) b > 2 e q um primo ou potência de um primo;

b) $b=1$ e q um primo ímpar ou potência de um primo (conjunto de corte arbitrário) 


$$
d_{\min } \leqslant\left[\frac{q^{p^{*}-1}}{q^{p^{*}}-1}\right] m \varphi
$$

onde $\mathrm{p}^{*}$ é o inteiro positivo que minimiza o termo à direita em cada uma das inequações acima.

É interessante comentar que o Teorema 2 estabelece um conjunto de soluções bastante reduzido de $\mathbf{P}_{1}$. Notamos também que, em geral, a distância mínima, $\mathrm{d}_{\min }$, iguala-se à distância livre, $\mathrm{d}_{\text {free}}$, dos códigos convolucionais ótimos. Isto é, a menor distância mínima dos códigos convolucionais ótimos é alcançada já no primeiro comprimento de restrição $(m+1$ janelas de tempo na treliça).

Uma vez que a equivalência e redução ficaram estabelecidas, nosso próximo objetivo é explorar o emprego das técnicas de programação matemática no encaminhamento do algoritmo a ser proposto nas seções que se seguem. Estaremos voltados para a classe dos códigos convolucionais de memória unitária (que abreviaremos para $\mathrm{CCMU}$ ), pois a mesma apresenta a propriedade surpreendente de que, para uma determinada multiplicidade de taxa, a distância livre deste cód go pode ser superior à distância livre de qualquer outra classe de códigos convolucionais conhecidos [9] e [12].

Antes de passarmos efetivamente para a formulação do problema, definiremos código de memória unitária como aquele que satisfaz à seguinte relação:

$$
\mathbf{y}_{\mathrm{k}}=\mathrm{G}_{\mathrm{O}} \mathbf{u}_{\mathrm{k}} \oplus \mathbf{G}_{1} \mathbf{u}_{\mathrm{k}-1}
$$

onde $u_{k}=\left(u_{k 1}, u_{k 2}, \ldots, u_{k b}\right), y_{k}=\left(y_{k 1} y_{k 2}, \ldots, y_{k n}\right), k \leqslant 0$; com $u_{k}=0$ para $\mathrm{k}<0$, são vetores binários; $\mathbf{G}_{0} \mathbf{e ~}_{1}$ são as submatrizes da matriz geradora; $\mathbf{u}_{\mathrm{k}}$ representa um bloco de $b$ dígitos de informação a serem codificados no instante $\mathrm{k} ; \mathrm{y}_{\mathrm{k}}$ representa a correspondente palavra código-ramo, de comprimento $\mathrm{n}$, gerada no instante $\mathrm{k} e \oplus$ representa a adição em GF(2) (binário). Nota-se que o código é chamado de memória unitária porque apenas a informação relativa a um período anterior (que pode conter vários dígitos) é armazenda. Pode-se mostrar facilmente [9] que um codificador com várias memórias em série pode ser transformado, através do uso das memórias em paralelo, em um codificador de memória unitária. Para isto basta caracterizar as matrizes $\mathbf{G}_{0}$ e $\mathbf{G}_{\mathbf{1}}$, do $\mathrm{CCMU}$, como sendo as matrizes com dimensão $(\mathrm{bm}) \times(\mathrm{nm})$ formadas a partir das matrizes bxn do código convolucional original. A transformação inversa, isto $e$, do CCMU para um código convolucional com memória $m>1$ nem sempre é possível. Em conseqüência, pode-se afirmar que os códigos de memória múltipla formam um subconjunto da classe dos códigos de memória unitária. 


\section{Resoluçāo por Programaçāo Matemåtica}

A análise das características de um CCMU através do diagrama de transição de estado é bastante facilitada porque este diagrama contém toda a informação necessária para se avaliar o desempenho do código. Assim, o uso desta representação é interessante quando são dadas as matrizes geradoras e se deseja avaliar a capacidade de correção do código correspondente, o que ocorre quando se procura determinar códigos ótimos através de algoritmos de enumeração extensiva (implícita ou explícita) das matrizes geradoras. Contudo, estes algoritmos de enumeração extensiva têm sua aplicação limitada pelo crescimento exponencial das dimensões do problema. Uma altemativa é usar algoritmos heurísticos que procuram obter uma solução ótima, ou próxima da ótima, melhorando gradativamente uma solução disponivel. Veremos agora como uma análise de outros tipos de representação dos CCMUs pode levar à idealizaçāo de métodos eficientes de busca de códigos ótimos. Inicialmente, devemos considerar como os códigos de bloco estấo relacionados com os CCMUs. Como um código convolucional é linear, sua capacidade de correção depende das distâncias de Hamming entre as palavras código, que são iguais aos pesos de Hamming das palavras não nulas. Por isto, nos parágrafos seguintes usaremos igualmente os termos peso e distância de Hamming, implicitamente excluindo a palavra código nula.

Seja $d^{*}$ free a máxima distância livre dos códigos $(n, b, 1)$. Para que um CCMU especiffico tenha distância livre igual a $d^{*}$ free é necessário que todas as palavras código truncadas, com comprimento $2 n, 3 n, . .$. , tenham peso de Hamming maior que ou igual a $d^{\star}$ free. Isto significa que as matrizes geradoras $\mathbf{G}_{0}$ e $\mathbf{G}_{1}$ devem ser tais que o peso mínimo das palavras dos códigos de bloco gerados pelas matrizes apresentadas em (4) sejam maiores que ou iguais a $d^{\star}{ }_{\text {free. }}$

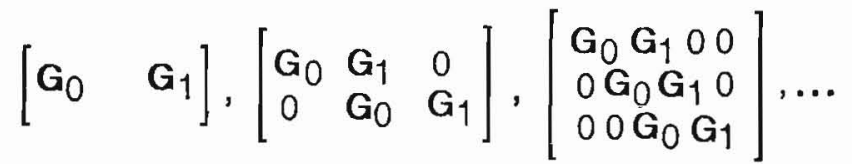

Esta condição pode ser usada para calcular limitantes superiores de $d^{*}$ free. Seja $\delta(n, b)$ o valor máximo do peso mínimo das palavras de um código de bloco $(n, b)$. Podemos então limitar $d^{*}$ free por [9] e [12]

$$
d_{\text {free }}^{*} \leqslant \delta([p+1] n, p b), \quad p=1,2, \ldots
$$

Esta representação por uma seqüência de códigos de blocos, que é relacionada com a representação das transiçōes de estado por uma árvore, não é muito utilizada pela dificuldade de se considerar explicitamente todas as palavras 
código de comprimentos múltiplos de n. Contudo, analisando (5) é fácil concluir que, se quisermos que o $\mathrm{CCMU}$ gerado por $\mathrm{G}_{0}$ e $\mathrm{G}_{1}$ tenha distância livre igual a $\mathrm{d}^{*}$ free, uma condição necessária, mas não suficiente, é que o peso minimo do código de bloco gerado por $\left[\mathbf{G}_{0} \mathbf{G}_{1}\right]$ seja maior ou igual a $d^{*}$ free. Além disto, para códigos com taxas não muito altas $(1 / 2 \leqslant r \leqslant 3 / 4)$, a expe riência prática demonstra que, nos códigos ótimos, a distância livre é determinada pela distância entre as palavras geradas pela matriz $\left[\mathbf{G}_{0} \mathbf{G}_{1}\right]$, que têm comprimento $2 n$, já que as palavras geradas pelas outras matrizes têm comprimento $3 n, 4 n, . .$. , e portanto devem ter um peso maior, dependendo do número de bits redundantes ( $n$-b). No diagrama de transição de estado, esta suposição é equivalente a se considerar que os "caminhos" de menor peso de Hamming são os que saem do estado $\mathbf{0}$, passam por apenas um estado intermediário e voltam imediatamente ao estado 0 .

A distância de Hamming entre as palavras código geradas pela matriz $\left[\mathbf{G}_{0} \mathbf{G}_{1}\right]$ depende apenas da configuração binária (tipos) de suas colunas e não da ordem destas colunas na matriz, já que esta corresponde a uma matriz geradora de um código de bloco linear e, para estes códigos, se trocarmos de posição as colunas, alteraremos as palavras código mas não o seu peso de Hamming. No caso das matrizes geradoras das palavras de comprimento maior que $2 n$ que aparecem em (4), esta ordem é importante para determinar a distância entre as palavras código devido ao interrelacionamento entre as submatrizes $\mathrm{G}_{0}$ e $G_{1}$ na formação de suas colunas.

Estas propriedades, que foram mais facilmente visualizadas por esta representação do CCMU por códigos de bloco, são suficientes para se propor a decomposição do problema de determinação do código ótimo em dois subproblemas menores e de resolução mais simples. A estratégia de decomposição a ser empregada objetiva eliminar a necessidade de testar um número muito grande de soluções. As duas etapas foram definidas conforme descrito a seguir.

\section{Processo de Seleção dos Tipos de Coluna da Matriz Geradora}

Determinar um conjunto de $2 n$ colunas tais que, se usadas para formar $\left[\mathrm{G}_{0} \mathrm{G}_{1}\right]$, independentemente da sua ordenção, maximizam o peso mínimo do código de bloco gerado por $\left[\mathbf{G}_{0} \mathbf{G}_{1}\right]$. Como vimos anteriormente, esta etapa define uma condição necessária e portanto o peso destas palavras deve sermaior ou igual a $\mathrm{d}^{*}$ free. Nesta etapa as matrizes $\mathbf{G}_{0}$ e $\mathbf{G}_{1}$ são analisadas como em um código de bloco.

\section{Processo de Alocação dos Tipos nas Colunas da Matriz Geradora}

A partir do conjunto de tipos de colunas obtido pelo processo anterior, determi- 
nar que alocação destes tipos nas colunas de $\mathbf{G}_{0}$ e $\mathbf{G}_{1}$ formará uma matriz geradora cujo código convolucional tem distância livre igual a $d^{*}$ free. Pode-se notar que apenas nesta etapa irá se considerar $\mathbf{G}_{0}$ e $\mathbf{G}_{1}$ como matrizes geradoras de um código convolucional.

O ponto fundamental na aplicação eficiente desta estratégia de decomposição é a conjetura de que, ao se avançar da primeira para a segunda etapa, cada conjunto de soluções que não é eliminado conterá, com grande probabilidade, soluções ótimas. Na prática, isto significa que podemos analisar um pequeno número de soluções do problema de seleção de tipos de colunas (ao invés de todas) e, neste conjunto, encontrar uma soluçāo ótima do problema original através do algoritmo de alocação. Com esta condição, é possivel a aplicação de algoritmos heurísticos de busca bastante eficientes na resolução dos subproblemas, conforme veremos nas seções 4 e 5 . Inicialmente pode-se considerar que esta conjetura aplica-se apenas aos códigos de taxa mais baixa ( $r$ $\leqslant 1 / 2$ ), onde o maior número de bits redundantes conduz a problemas com mais graus de liberdade. Contudo, resultados práticos mostram que esta conjetura pode ser válida mesmo para taxas altas, como $r=7 / 9$.

\section{Determinação das Colunas da Matriz Geradora}

Como vimos anteriormente, pode-se formular um algoritmo de busca de códigos convolucionais ótimos a partir de códigos de bloco, mas os códigos de bloco normalmente usados, que são sistemáticos, não são os mais indicados para formar códigos convolucionais ótimos. É necessária, então, uma definição matemática do problema, a partir da qual possa ser criado um algoritmo capaz de gerar um conjunto relativamente grande de códigos não sistemáticos, satisfazendo às condições de distância apresentadas na Seção 3.

A determinação de um código de bloco linear $(2 n, b)$ gerado por uma matriz bjnária $\mathbf{G}=\left[\mathbf{G}_{0} \mathbf{G}_{1}\right]$ e com máxima distância mínima de Hamming (capacidade de correção) pode ser formulada como um problema de otimização. Neste caso, é interessante que esta formulação tenha certas propriedades como linearidade e convexidade, o que é dificultado pelo fato das operaçōes de geração do código, com a matriz G, serem definidas em GF(2) (operações binárias). Para contornar esta dificuldade, pode-se usar o artifício de construir uma tabela com os resulados das operações binárias, como veremos a seguir.

Inicialmente define-se um vetor de variáveis de problema $c_{\text {, tal }}$ que $c_{i}$ $=\mathrm{r}$ se $\mathrm{r}$ tipos de colunas de $\mathrm{G}$ tiverem representação binária igual a i. As colunas com todos os bits iguais a 0 não são representadas e $\mathbf{c}$ tem dimensão $\mathrm{N}=$ $2^{b}-1$. A seguir, definimos uma matriz $\mathrm{M}_{\mathrm{b}}$, com dimensão $\mathrm{N} \times \mathrm{N}$, que contém to- 
dos os possíveis valores não nulos dos vetores binários de dimensão b. Por exemplo, se $b=3$, temos

$$
\mathbf{M}_{3}=\left[\begin{array}{lllllll}
0 & 0 & 0 & 1 & 1 & 1 & 1 \\
0 & 1 & 1 & 0 & 0 & 1 & 1 \\
1 & 0 & 1 & 0 & 1 & 0 & 1
\end{array}\right]
$$

Podemos notar que a matriz $\mathrm{M}_{\mathrm{b}}$ contém todos os possiveis tipos de colunas de $\mathbf{G}$ e $\mathbf{M}_{b}^{t}$ (sua transposta) contém todos os possiveis vetores binários não nulos de entrada do codificador. Desta forma, se definirmos $\mathrm{H}_{b}=$ $\mathrm{M}_{\mathrm{b}} \mathrm{t} \otimes \mathrm{M}_{\mathrm{b}}$, onde a operação entre as matrizes é feita em $\mathrm{GF}(2), \mathrm{H}_{\mathrm{b}}$ irá conter todos os resultados binários dos produtos de todos os possíveis tipos de colunas da matriz $\mathbf{G}$ por todos os possíveis vetores binários de entrada. O peso de Hamming das palavras código correspondentes pode ser calculado somando os termos das linhas de $\mathrm{H}_{\mathrm{b}}$ correspondentes às colunas pertencentes a G. Portanto, podemos calcular o vetor de pesos das palavras código, d. através de

$$
d=H_{b} c
$$

Com estas definições, todas as operações binárias são feitas no produto $M_{b}{ }^{t} \otimes$ $\mathbf{M}_{\mathrm{b}}$ e as propriedades de distância do código, gerado pela matriz $\mathbf{G}$, podem ficar implícitas em (6). A representação da matriz $\mathbf{G}$ pelo vetor de variáveis $\mathbf{c}$ já inclui a propriedade de que a ordem das colunas não irá alterar os pesos das palavras código. Note que (6) justifica a escolha da represetação das matrizes geradoras pelas suas colunas.

Chegamos então a uma forma de calcular os pesos de Hamming das palavras código que não envolve operações binárias diretamente, que é linear, mas com a restrição de que os elementos do vetor $\mathbf{c}$ sejam inteiros. Como, normalmente, temos um grande número de códigos cuja distância mínima é igual a $\mathrm{d}^{*}{ }_{\text {free}}$, para minimizar a probabilidade de erro, é interessante minimizar também o número de palavras código que têm peso igual a $\mathrm{d}^{\star}$ free. Com este intento, definimos uma função de penalização da forma 


$$
\pi(x, y)=\left\{\begin{array}{l}
(y-x)^{2} \text { se } y \geqslant x \\
0 \text { caso contrårio }
\end{array}\right.
$$

Podemos então formular o problema $P_{2}$ como:

$$
\begin{array}{ll}
\mathbf{P}_{2} \quad \text { Minimizar } & z(\mathbf{c})=\sum_{i=1}^{N} \pi\left(d_{i}, d^{*} \text { free }^{+1}\right) \\
\text { sujeito a } & H_{b} \mathbf{c}=d \\
& \sum_{i=1}^{N} c_{i}=2 n \\
& c_{j} \in\{0,1,2, \ldots, 2 n\}
\end{array}
$$

Nesta formulação, a função objetivo em (7) é convexa e não linear, penalizando a ocorrência de palavras com peso menor que $\mathrm{d}_{\text {free. }}^{\star}$. Caso todas as palavras tenham peso maior ou igual $a d^{\star}$ free, a função objetivo minimiza o número de palavras com peso igual a $d^{*}$ free. $A$ restrição em (8) representa apenas uma transformação de variáveis para simplificar a notação e dé o vetor com os pesos de Hamming das palavras código. A restrição em (9) faz com que a quantidade dos tipos de colunas seja igual ao número de colunas da matriz geradora, que, pela restrição em (10), deve ser um número inteiro. Como (8) é apenas uma mudança de variável, podemos notar que as restrições em (9) e (10) definem o chamado problema de seleçāo, ou seja, selecionar um conjunto de 2n tipos de colunas que irá maximizar uma função avaliadora da capacidade de correção do código.

$\mathrm{O}$ problema $\mathrm{P}_{2}$ é um problema de otimização com variáveis inteiras, que pode ser resolvido por várias ferramentas de programação matemática [13]. As considerações sobre a escolha do método mais apropriado e sua descrição seriam muito extensas para serem incluídas neste trabalho. Em [14] é apresentado um algoritmo heurístico desenvolvido especialmente para resolução deste problema.

\section{Ordenação das Colunas da Matriz Geradora}

A partir das soluções do problema $P_{2}$, temos uma determinada quantidade de conjuntos de tipos de colunas que, se usadas para formar $\mathbf{G}_{0}$ e $\mathbf{G}_{1}$, satisfazem a condição necessária de que nenhuma palavra do código convolucional de tamanho $2 n$ terá peso menor que $\mathrm{d}^{*}$ free. Além disso, pelo que foi concluído na Seção 3, existe uma grande probabilidade de que algumas das ordenaçōes 
destas colunas irão gerar códigos convolucionais não catastróficos com distância livre igual a $d_{\text {free }}^{*}$ e, dentre estas, devemos ter aquela ordenação cujo código têm a menor probabilidade de erro.

Para representação das variąveis do problema, devemos considerar que temos $2 n$ colunas de $G_{0}$ e $G_{1}$ que podem ser colocadas em $2 n$ posições. Trata-se, portanto, de um clássico problema de alocação, o que sugere representar as variáveis pela matriz $P$, de dimensão $2 n \times 2 n$, tal que $p_{i j}=1$ se o tipo i está na posição j e $p_{i j}=0$ se não está. $\mathbf{A}$ matriz $\mathbf{P}$ também pode ser interpretada como uma matriz de permutação aplicada a uma matriz $\mathrm{G}$ que tem uma ordenação arbitrária dos tipos de colunas determinadas pelo problema $\mathbf{P}_{2}$.

Este problema de alocação também pode ser formulado como um problema de otimização, mas o interrelacionamento entre $\mathbf{G}_{0}$ e $\mathbf{G}_{1}$ na formação das palavras do código convolucional dificulta a eliminação das operações binárias na formulação do problema de otimização correspondente. O artifício utilizado na formulação do problema $P_{2}$ pode se usado, se considerarmos as posições dos tipos de $G_{0}$ ou de $G_{1}$ fixas. Neste caso, é necessario ponderar a eficiência na resolução versus a fidelidade na formulação. Neste trabalho, escolhemos a primeira opção para poder determinar códigos de maior dimensão.

A segunda etapa para formulação matemática do problema é a escolha da representação mais apropriada do código. Usando, por exemplo, a representação por códigos de blocos, considerando as palavras código de comprimento 3 , temos o seguinte problema:

$P_{3}$

$$
\text { Minimizar } \quad w(P)=\sum_{l=1}^{2 b_{-1}} \pi\left(\mathrm{d}_{l}, \mathrm{~d}_{\mathrm{g}}+1\right)
$$

sujeito a

$$
\begin{aligned}
& \sum_{i=1}^{n} p_{i j}=1, \quad j \in\{1,2, \ldots, n\} \\
& \sum_{j=1}^{n} p_{i j}=1, \quad i \in\{1,2, \ldots, n\} \\
& p_{i j} \in\{0,1\} \\
& d_{i}=\sum_{i=1}^{n} \sum_{j=1}^{n} h_{i j l} \quad p_{i j}, \quad \mid \in\left\{1,2, \ldots 2^{2 b-1\}}\right.
\end{aligned}
$$


A função objetivo é semelhante à do problema $P_{2}$, mas usando uma distância

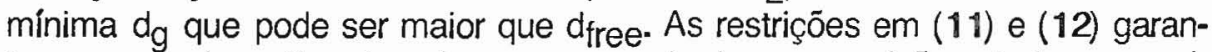
tem que nenhum tipo de coluna ocupa mais de uma posição e todas as posições são ocupadas. As restrições em (14) representam apenas uma mudança de variável, definindo os pesos das palavras código. Os parâmetros $h_{i j l}$ dão o incremento no peso da palavra código I, caso o tipo i esteja na posição j.

A grande dificuldade na resolução deste problema a sua grande dimensão, que é proporcional a $2^{2 b}$. Por exemplo, para um código $(12,7)$ temos aproximadamente dezesseis mil variáveis $d_{j}$ e mais de dois milhões de valores de $h_{i j l}$ que devem ser calculados e armazenados. Além disto, como as soluções de $\mathbf{P}_{3}$ satisfazem apenas mais uma condição necessária, é preciso considerar também as palavras código com comprimento $3 n, 4 n, \ldots$, que produzem problemas com dimensão muito maior.

Para ser possível trabalhar com palavras código de comprimento arbitrårio é preciso voltar a uma representação mais compacta e um algoritmo de análise que permita selecionar apenas as palavras de mínimo peso, que são as mais importantes na avaliação da capacidade de correção. Podemos usar as propriedades definidas na Seção 2, e incluir (14) na formulação de $\mathbf{P}_{1}$ (o problema de máximo fluxo). Neste caso, temos um interrelacionamento entre os problemas de alocação de recursos e máximo fluxo, podendo-se usar um algoritmo de programação matemática que, a partir da resolução sequencial dos dois problemas, chegue ao código ótimo.

Podemos ainda usar um método heurístico para solucionar o problema de alocação, considerando empiricamente duas propriedades dos CCMUs. Primeiramente, devemos levar em conta que se alterarmos apenas dois valores de $\mathrm{P}$, mantendo a factibilidade em relação às restrições (11) até (13), a máxima variação dos pesos das palavras código de comprimento $x n, x>2$, é igual $\mathrm{a} \pm 2(\mathrm{x}-2)$. Esta "pequena alteração" é equivalente a trocar duas colunas de posição. A segunda propriedade é que, normalmente, para códigos com taxa $r \geqslant 1 / 2,0$ perfil das distâncias ("distance profile") apresenta um crescimento rápido dos pesos de Hamming das respectivas palavras código.

A partir destas propriedades podemos esperar que pequenas alteraçōes nas variáveis $\mathrm{p}_{\mathrm{ij}}$ produzem pequenas variaçōes nas propriedades do código, ou seja, as variações na função avaliadora de um CCMU devem ser "suaves" em relação a pequenas variações em $\mathbf{P}$. Assim, podemos também esperar que um algoritmo que vá cumulativamente melhorando as características do código seja viável. Contudo, trata-se de um problema bem mais complexo do que $\mathrm{P}_{2}$, o que deve dificultar este processo de busca localizada. 
A grande liberdade que se obtêm através do uso de um algoritmo heuristico permite tentar minimizar também o número de palavras com peso mínimo, o que pode ser conseguido pela aplicação do algoritmo de Viterbi. Mais uma vez, é ịnteressante mudar a representação do código para analisar as variaçöes nas palavras de peso mínimo, usando a representação por treliça. Na Fig. 2 temos a representação de uma treliça em 5 janelas de tempo. Podemos definir uma função de avaliação do código a partir dos resultados da aplicação do algoritmo de Viterbi. A idéia é fixar um conjunto de parâmetros $d(r){ }_{m}$, iguais ou maiores que os valores mínimos esperados dos pesos das palavras de comprimento r. Se, durante a aplicação do algoritmo de Viterbi, for encontrada uma palavra com peso $\delta$, menor que $d(r) m$, a função de penalização $\pi(\delta, d(r) m)$ anteriormente definida é somada à função de avaliação do código. $O$ algoritmo heuristico consiste em trocar as colunas da matriz geradora, duas a duas, cada vez que isto acarreta uma diminuição na função de penalização. Em (14) é apresentada de uma descrição deste algoritmo.

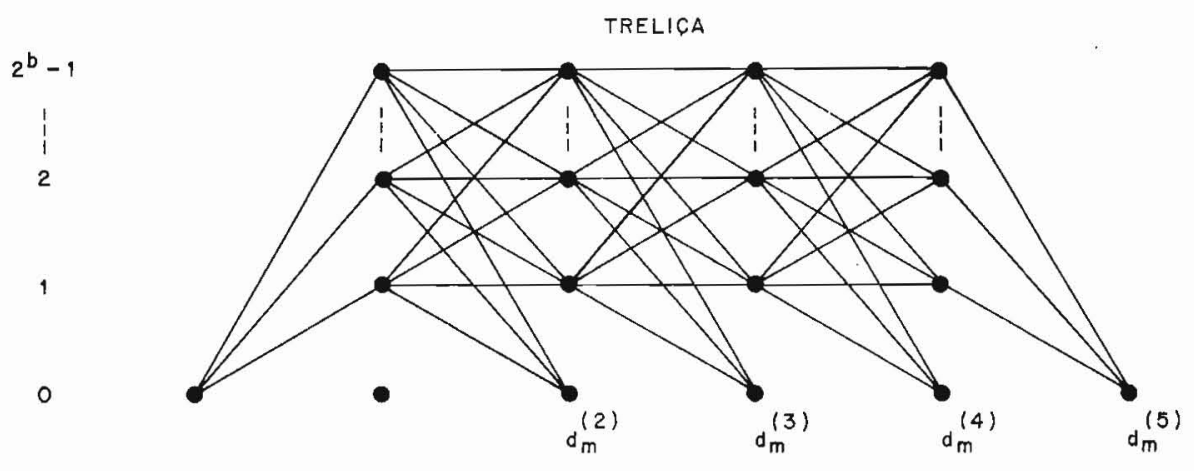

Figura 2. Diagrama de treliça.

\section{Resultados Computacionais}

Conforme explicado na Seção 3, onde se justifica o processo de decomposição, uma condição esperada para o sucesso da aplicação dos algoritmos de resolução dos problemas $\mathbf{P}_{2}$ e $\mathbf{P}_{3}$ é que os códigos procurados não tenham taxas muito altas. Os melhores resultados são esperados para taxas menores que $1 / 2$, quando os critérios de otimalidade podem ser mais facilmente alcançados pela existência de um número suficiente de bits redundantes, de modo que o perfil de distâncias cresce rapidamente. Contudo, foi possível obter bons resultados mesmo para taxas maiores que $3 / 4$, conforme a dimensão do problema. Neste último caso, o preço a pagar foi um menor controle da distribuição dos pesos das palavras, embora conseguindo-se códigos com distância li- 
vre igual ao máximo possivel (o que, para alguns autores, já representa a otimalidade do código).

$\mathrm{Na}$ Tabela 2 são apresentados alguns códigos convolucionais de memória unitária ótimos, até então não conhecidos na literatura técnica. $\mathrm{Na}$ obtenção destes códigos, os seguintes critérios de otimalidade foram empregados (em prioridades crescente):

(i) eliminação dos códigos catastróficos;

(ii) maximização de $d_{\text {free}}$,

(iii) minimização do número de palavras código com peso $d_{\text {free; }}$

(iv) crescimento dos pesos das palavras código na treliça.

É interessante observar que ao se incluir o critério de crescimento do peso das palavras na treliça para selecionar os códigos com máxima distância livre, é possivel eliminar os códigos catastróficos, já que estes têm um perfil de distância inferior. Não chegamos a encontrar uma classe de códigos $(n, b)$, com $r \geqslant 2 / 3$ e $b>4$, em que todos os códigos com máximo dfree são catastróficos.

Todos os cớdigos na Tabela 2 têm b e n primos entre si e portanto não podem ser transformados em códigos de memória não unitária. Para se avaliar seu desempenho, são apresentados os ganhos assintóticos $(\gamma)$ de cada código para o canal AWGN ("Additive White Gaussian Noise"), sendo $\gamma$ para um código $(n, b, m)$, com distância livre $d_{\text {free }}$ e $2^{m}$ estados, calculado por

$$
\gamma=10 \log _{10} \frac{b d_{\text {free }}}{2 n} \quad d B
$$

Os valores de $\gamma$ obtidos podem ser comparados com ganhos assintóticos de outros códigos ótimos, de memória múltipla, apresentados em [15]. Por exemplo, o novo código de memória unitária $(7,5,1)$ apresenta a mesma distância livre que o código $(3,2,3)$ ótimo de [5], porém, quando comparados seus desempenhos vemos que o código $(7,5,1)$ apresenta um valor de $\alpha$ igual a 3,31 enquanto que o código $(3,2,3)$ apresenta um valor de $\gamma$ igual a 3,10. Portanto, pode-se concluir que o novo código tem um melhor desempenho com uma complexidade equivalente. 


\begin{tabular}{|c|c|c|c|c|c|}
\hline$b$ & $n$ & $\mathrm{G}_{0}$ & $\mathrm{G}_{1}$ & $d_{\text {free }}$ & $\gamma(\mathrm{dB})$ \\
\hline 5 & 6 & $\begin{array}{l}000011 \\
100010 \\
011000 \\
011110 \\
010010\end{array}$ & $\begin{array}{l}011011 \\
110111 \\
100100 \\
001010 \\
001101\end{array}$ & 4 & 2,22 \\
\hline 5 & 7 & $\begin{array}{l}0110001 \\
0100100 \\
0011011 \\
1100000 \\
0000011\end{array}$ & $\begin{array}{l}0010110 \\
1010101 \\
1011100 \\
0101011 \\
0111010\end{array}$ & 6 & 3,31 \\
\hline 5 & 8 & $\begin{array}{l}00110111 \\
01100010 \\
11100101 \\
00100100 \\
01011000\end{array}$ & $\begin{array}{l}11010010 \\
01011111 \\
00010110 \\
11101110 \\
10110110\end{array}$ & 7 & 3,40 \\
\hline 6 & 11 & $\begin{array}{l}11101011101 \\
11001001000 \\
10011100110 \\
10110010110 \\
10000001110 \\
00111000010\end{array}$ & $\begin{array}{l}10101110100 \\
11100010001 \\
10100010100 \\
00000111010 \\
11101100110 \\
11010100100\end{array}$ & 9 & 3,90 \\
\hline 6 & 13 & $\begin{array}{l}0001110100000 \\
1100110001100 \\
0010101110011 \\
0100010110001 \\
0000111001001 \\
0000101100110\end{array}$ & $\begin{array}{l}1111001110001 \\
0100011111000 \\
0000100111010 \\
1001011101010 \\
0011100010111 \\
1100011001011\end{array}$ & 12 & 4,42 \\
\hline 7 & 9 & $\begin{array}{l}110100010 \\
001011100 \\
000100010 \\
100100100 \\
101010000 \\
100111100 \\
000101111\end{array}$ & $\begin{array}{l}110111010 \\
101101110 \\
110000011 \\
011001110 \\
100010010 \\
100010100 \\
000101000\end{array}$ & 6 & 3,68 \\
\hline
\end{tabular}

Tabela 2. Exemplos de códigos ótimos encontrados. 


\section{Conclusōes}

Neste trabalho foi apresentado um procedimento direcionado à sistematização do problema de seleção de códigos convolucionais de memória unitária via técnicas de otimização, em contraposição às técnicas de busca exaustivas até então utilizadas. A determinação da matriz geradora do código foi possível graças à resolução de dois problemas de otimização inteira, cuja função objetivo penaliza as palavras código com peso de Hamming abaixo do desejado. $O$ primeiro problema seleciona os tipos de colunas que formarão a matriz geradora. A determinação da ordem das colunas nas submatrizes geradoras foi conseguida após a resolução de um problema de alocação de recursos. Como resultado desta nova metodologia, novos códigos convolucionais de memória unitåria foram apresentados.

\section{Referências}

[1] P. Elias, "Coding for Noisy Channels", IRE Convention Record, Part 4, 1955, pp.37-47.

[2] A.J. Viterbi, "Error Bounds for Convolutional Codes and an Asymptotically Optimum Decoding Algorithm", IEEE Transactions on Information Theory, vol. IT-13, no. 2, Abril 1967, pp. 260-269.

[3] J.P. Odenwalder, "Optimal Decoding of Convolutional Codes", PhD Dissertation, University of California, Los Angeles, 1970.

[4] K.J. Larsen, "Short Convolutional Codes with Maximum Free Distance for Rates 1/2, 1/3 and 1/4", IEEE Transactions on Information Theory, vol. IT19, no. 3, Maio 1973, pp. 371-372.

[5] E. Paaske, "Short Binary Convolutional Codes with Maximal Free Distance for Rates 2/3 and 3/4", IEEE Transactions on Information Theory, vol. IT-20, no. 5, Setembro 1974, pp. 683-698.

[6] R. Johannesson e E. Paaske, "Further Results on Binary Convolutional Codes with an Optimum Distance Profile", IEEE Transactions on information Theory, vol. IT-24, no. 2, Março 1978, pp. 264-268.

[7] J.L. Massey e D.J. Costello, Jr., "Nonsystematic Convolutional Codes for Sequential Decoding in Space Applications", IEEE Transactions on Communication Technology, vol. COM-19, no. 5, Outubro 1971, pp. 806-813. 
[8] D.G. Daut, J.W. Modestino e L.D. Wismer, "New Short Constraint Lenght Convolutional Code Constructions for Selected Rational Rates", IEEE Transactions on Information Theory, vol. IT-28, no. 5, Setembro 1982, pp. 794-800.

[9] L.N. Lee, "Short Unit-Mernory Byte Oriented Binary Convolutional Codes Having Maximal Free Distance", IEEE Transactions on Information Theory, vol. IT-22, no. 3, Maio 1976, pp. 349-352.

[10] R. Palazzo Jr., "Analysis of Periodic Linear and Nonlinear Trellis Codes", PhD Dissertation, University of California, Los Angeles, 1983.

[11] R. Palazzo Jr., "A General Relationship Between the Maximum Flow in Networks and the Minimum Distance of Convolutional Codes Over GF(q)", 3rd Joint USSR-Swedish International Workshop on Information Theory, Sochi, USSR, Maio 1987.

[12] G. Thommesen e J. Justesen, "Bounds on Distance and Error Exponents of Unit Memory Codes", IEEE Transactions on Information Theory, vol. IT29, no. 5, Setembro 1983, pp. 637-649.

[13] G.L. Nemhauser e L.A. Wolsey, "Integer and Combinatorial Optimization", John Wiley \& Sons, 1988.

[14] A. Said e R. Palazzo Jr., "Heuristic Search: A New Method to Find Good Unit-Memory Convolutional Codes", Fourth Joint Swedish-Soviet International Workshop on Information Theory, Gotland, Suécia, Agosto 1989.

[15] S. Lin e D. J. Costello Jr., "Error Control Coding: Fundamentals and Applications", Prentice-Hall, Englewood Cliffs, N.J., 1983.

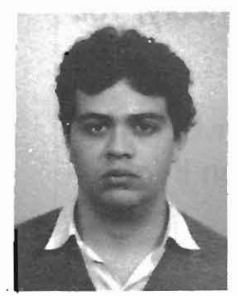

AMIR SAID formou-se em Engenharia Elétrica pela UNICAMP em 1985, obteve o título de Mestre em Engenharia Elétrica pela UNICAMP em 1988. Desde 1987 atua como Professor da Faculdade de Engenharia Elétrica da UNICAMP. Ocupou o cargo de Engenheiro associado junto à IBM do Brasil onde de maio a novembro de 1986 participou do grupo de desenvolvimento de um equipamento periférico nos Laboratórios da IBM em Tucson, Arizona e Boca Raton, Flórida. Atualmente encontra-se realizando estudos no Rensselaer Polytechnic Institute, Troy, New York para obtenção do título de Ph.D. 


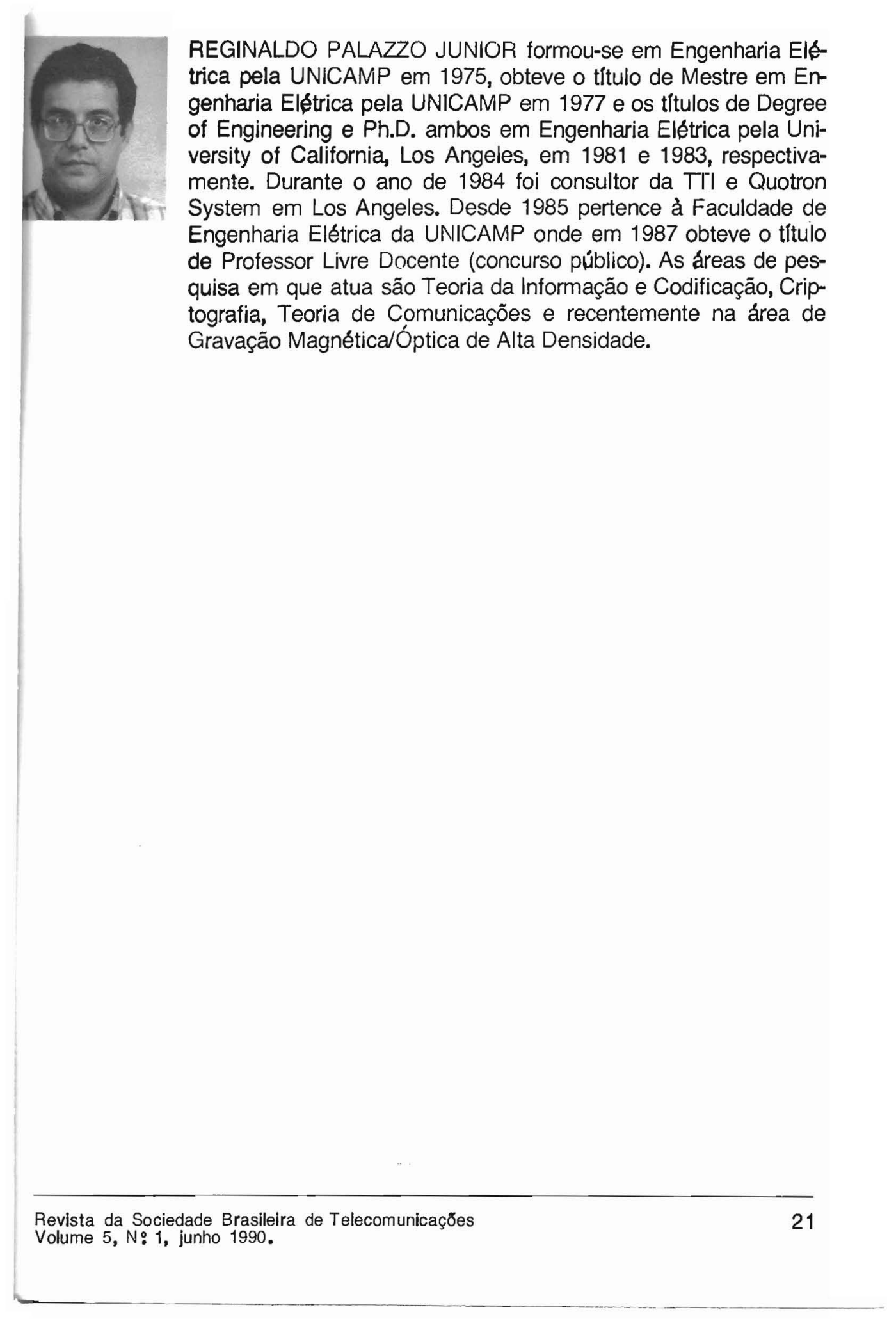

